\title{
Late onset endophthalmitis associated with intraocular lens: a case of molecularly proved $S$. epidermidis aetiology
}

\author{
B Jansen, C Hartmann, F Schumacher-Perdreau, G Peters
}

University of Cologne, Germany Institute of Medical Microbiology and

Hygiene

B Jansen

F Schumacher-Perdreau

G Peters

\section{Eye Clinic}

Correspondence to:

Dr B Jansen, Institute of

Medical Microbiology and

Hygiene, Goldenfelsstrasse 19-21, 5000 Cologne 41, Germany.

Accepted for publication 6 December 1990
C Hartmann

\begin{abstract}
A case of severe endophthalmitis after cataract extraction followed by posterior chamber lens implantation is reported. Microbiological cultures from a tap of the patient's aqueous humour prior to lens explantation as well as from the explanted lens and aqueous and vitreous humour during operation yielded Staphylococcus epidermidis sensu stricto. Scanning electron microscopy showed massive colonisation of the lens loop by staphylococci. Clonal identity of all isolates was demonstrated by plasmid DNA analysis and sodium dodecyl sulphate-polyacrylamide gel electrophoresis (SDS-PAGE) of extracellular products. This is strongly suggestive of the aetiological role of $S$. epidermidis in this case of late onset endophthalmitis.
\end{abstract}

Coagulase-negative staphylococci - in particular $S$. epidermidis sensu stricto - are the commonest organisms isolated from foreign body infections. ${ }^{1}$ The pathogenesis of these infections is characterised by the ability of these bacteria to adhere to and grow on polymer surfaces and to produce an extracellular slime substance which

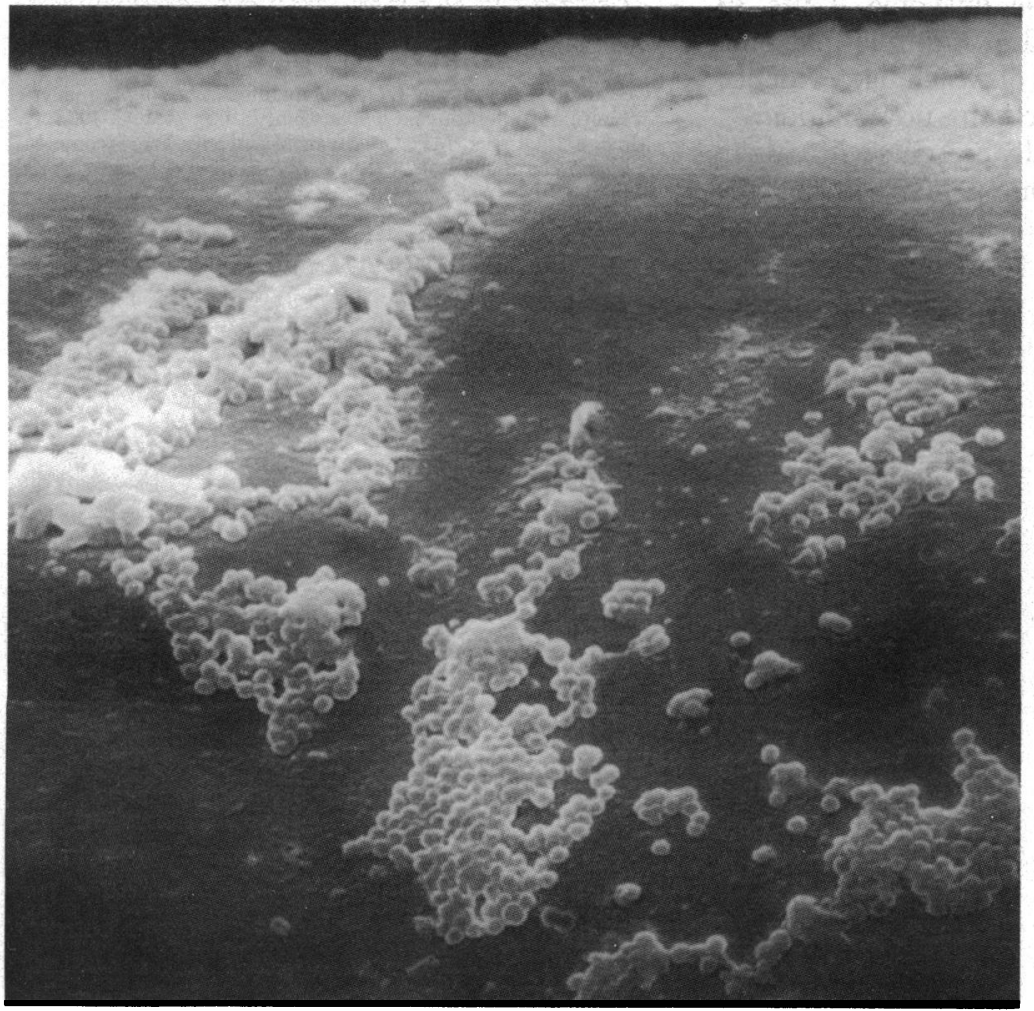

Figure 1 Scanning electron micrographs of the lens loop after explantation. A massive colonisation by staphylococcal cells can be seen. protects the embedded bacterial cells against the host's immune response and antibiotic therapy. ${ }^{23}$ Moreover, $S$. epidermidis is regarded as one of the commonest micro-organisms isolated in late onset endophthalmitis. ${ }^{4}$ However, until now its aetiological role in these infections has not been proved, mainly because of the lack of an adequate animal model.

Here we report a clinical case in which a patient developed a recurrent endophthalmitis after intraocular lens implantation. The $S$. epidermidis strains isolated from the anterior chamber before surgery and isolates from the infected lens, aqueous humour, and anterior chamber after surgery showed clonal identity as determined by molecular methods.

\section{Case report}

An 81-year-old man underwent an extracapsular cataract extraction followed by posterior chamber lens implantation in July 1987 in another hospital. On the third day after operation inflammation of the eye with fibrin deposition and hypopyon were diagnosed. The posterior segment was also affected, showing massive vitreous infiltrates; in addition there was a transient corneal oedema. On treatment with steroids and antibiotics (substances and doses not known) all inflammatory signs slowly regressed. Nevertheless, there was continuous inflammation of the entire eye.

In January 1988 the patient was seen in our clinic with severe endophthalmitis and treated again with antibiotics (cefotaxime $2 \mathrm{~g}$ three times a day, gentamicin $40 \mathrm{mg} / 40 \mathrm{mg} / 60 \mathrm{mg}$ intramuscularly per day), high doses of steroids (prednisolone $150 \mathrm{mg}$ per day), and topical subconjunctival injections of steroids and antibiotics. Paracentesis of the anterior chamber was performed and the aqueous humour submitted for culture and microscopical examination, as inflammation with hypopyon and vitreous infiltration persisted. Two weeks later the intraocular lens was explanted together with the capsular bag, and vitrectomy was performed. The lens, aqueous humour, and vitreous humour were examined by microbiological methods. In the three months following the second operation the inflammation slowly regressed. The final visual acuity was 0.5 in comparison with light perception and intact light projection before surgery.

Microbiological cultures from a tap of the patient's aqueous humour prior to lens explantation as well as from the explanted lens and aqueous and vitreous humour during the operation all yielded coagulase-negative staphylococci. 
Figure 2A Sodium dodecylsulphatepolyacrylamide gel electrophoresis (SDSPAGE) of crude extracellular products of the four isolates of S. epidermidis.

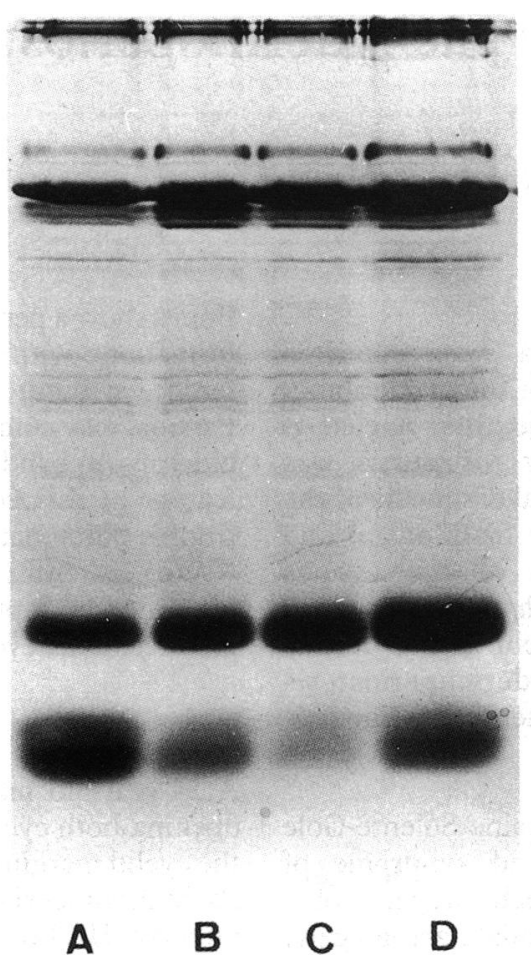

Scanning electron micrography of the lens loop (Prolene) showed massive colonisation by staphylococcal cells (Fig 1). Identification by the method of Kloos and Schleifer ${ }^{5}$ showed in all four cases $S$. epidermidis sensu stricto. The antimicrobial resistance pattern was also identical for the four isolates, showing resistance of the strains to penicillin, ampicillin, cotrimoxazole, and tetracycline. To demonstrate identity at the molecular level sodium dodecyl sulphate-polyacrylamide gel electrophoresis (SDS-PAGE, Fig 2A), and analysis of plasmid profiles (Fig 2B) were performed, showing identical patterns for the four isolates with both methods.

\section{Discussion}

There are a number of reports of intraocular infections presumably caused by $S$. epidermidis after intraocular lens implantation following cataract surgery. 6 In addition, the ability of $S$. epidermidis to adhere to and to colonise synthetic polymers has been demonstrated..$^{78}$ Recently, it was also shown that $S$. epidermidis can adhere to intraocular lenses. ${ }^{9}$ However, it has not yet been proved that it can cause this type of infection, mainly because of the lack of an adequate animal model. Furthermore, coagulase-negative staphylococci are normal colonisers of skin and mucous membranes and often found as contaminants in clinical material. The clonal identity of coagulase-negative staphylococcal strains obtained from different sites (such as infected lens, aqueous humour, anterior chamber) and at repeated intervals has not been demonstrated in any of the studies published so far.

In the case presented here we isolated $S$. epidermidis from the explanted lens and demonstrated by scanning electron microscopy

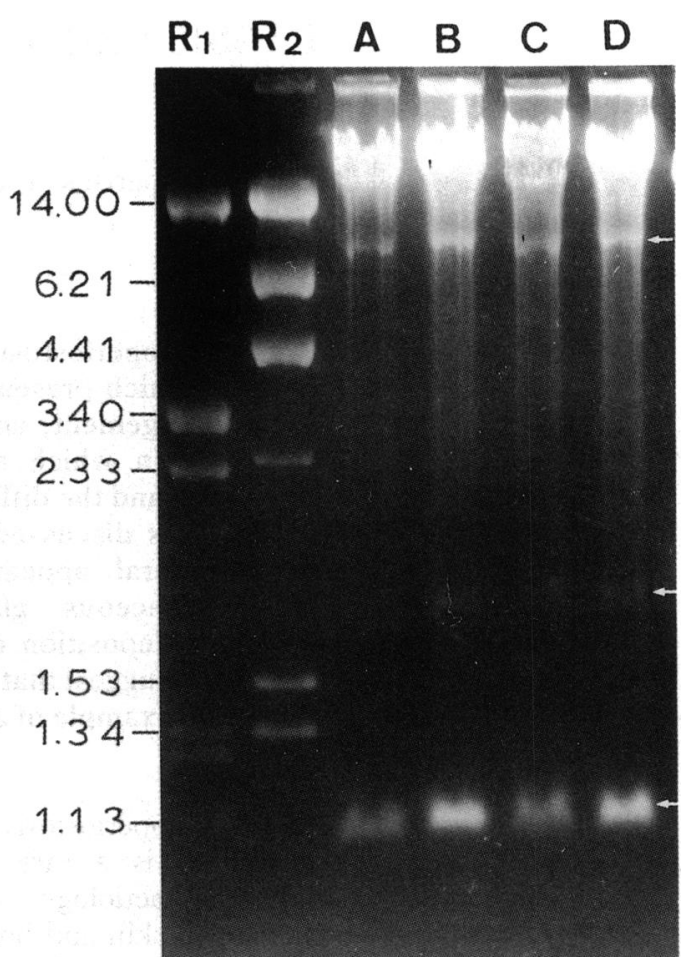

Figure 2B Agarose gel electrophoresis of plasmid DNA of the four isolates of S. epidermidis (molecular mass markers in megadaltons).

that staphylococcal cells were adherent to the lens loop embedded in a matrix of extracellular slime (Fig 1). All the $S$. epidermidis isolates cultivated from the lens, aqueous, and vitreous humour showed identity on the molecular level as shown by plasmid DNA analysis and SDSPAGE. To our knowledge this is the first report in which the clonal identity of $S$. epidermidis obtained from different sites and at repeated intervals could be demonstrated in a patient with intraocular infection after lens implantation. The findings are strongly suggestive of the aetiological role of $S$. epidermidis in this case of intraocular lens-associated late onset endophthalmitis. Thus this entity may be characterised as a chronic polymer-associated $S$. epidermidis infection.

1 Sugarman B, Young EJ. Infections associated with prosthetic devices. CRC Press, Boca Raton FL: 1984.

2 Peters G, Locci R, Pulverer G. Adherence and growth of coagulase-negative staphylococci on surfaces of intravenous catheters. F Infect Dis 1982; 146: 479-82.

3 Peters G, Pulverer G. Pathogenesis and management of Staphylococcus epidermidis 'plastic' foreign body infections. Staphylococcus epidermidis 'plastic' foreign body infe
$\mathcal{I}$ Antimicrob Chemother 1984; 14 (suppl D): 67-71.

4 Bohigan, GM, Olk RJ. Factors associated with a poor visual result in endophthalmitis. Am $\mathcal{F}$ Ophthalmol 1986; 101: 332-4.

5 Kloos WE, Schleifer KH. Simplified scheme for routine identification of human staphylococcus species. $\mathcal{F}$ Clin Microbiol 1975; 1: 82-8.

6 Weber DJ, Hoffman KL, Thoft RA, Baker AS. Endophthalmitis following intraocular lens implantation: report of $\mathbf{3 0}$ cases and literature review. Rev Infect Dis 1986; 8: 12-30.

7 Christensen GD, Simpson WA, Bisno AL, Beachey EH Adherence of slime producing stains of Staphylococcus epidermidis to smooth surfaces. Infect Immun 1982; 37: 318-26.

8 Ludwicka A, Jansen B, Wadström T, Switalski LM, Peters G, Pulverer G. Attachment of staphylococci to various polymers. In: Shalaby SW, Hoffman AS, Ratner BD, polymers. In: Shalaby SW, Hoffman AS, Ratner BD, Horbett TA, eds. P

9 Griffiths PG, Elliot TA, McTaggart L. Adherence of Staphylococcus epidermidis to intraocular lenses. Br $\mathcal{F}$ Ophthalmol
riffiths PG, Ellot TA, McTaggart L. Adherence of Staphy1989; 73: 402-6. 